\title{
Association of Chronic Obstructive Pulmonary Disease with Cognitive Decline in Very Elderly Men
}

\author{
Guoqing Zhou ${ }^{\mathrm{a}}$ Jinxia Liu ${ }^{\mathrm{c}}$ Fang Sun ${ }^{\mathrm{a}}$ Xiaofeng Xin ${ }^{\mathrm{b}}$ \\ Lihui Duan $^{\mathrm{a}}$ Xiaowei Zhu ${ }^{\mathrm{c}}$ Zhaorong Shi ${ }^{\mathrm{c}}$ \\ Departments of ${ }^{\mathrm{a}}$ Geriatric Neurology and ${ }^{\mathrm{b}}$ Respiratory Medicine, and $\mathrm{C}^{\mathrm{C}}$ Aging Clinical \\ Research Center, Jinling Hospital, Nanjing University School of Medicine, Nanjing, China
}

\section{Key Words}

Chronic obstructive pulmonary disease $\cdot$ Cognitive decline $\cdot$ Very elderly men $\cdot$ Dementia

\begin{abstract}
Aim: To determine the change in cognitive function in very elderly men with chronic obstructive pulmonary disease (COPD) over a 3-year period relative to age- and education-matched controls. Methods: In this hospital-based, prospective case-control study, we evaluated a consecutive series of 110 very elderly men with COPD and 110 control subjects who were hospitalized between January and December 2007. All the subjects performed cognitive tests at baseline and underwent annual evaluations (for 3 years), which included the Mini-Mental State Examination, word list recall, delayed recall, animal category fluency, and the symbol digit modalities test. Results: In mixed-effects models adjusted for hypertension and coronary heart disease, COPD was associated with a more rapid rate of cognitive decline based on the MiniMental State Examination, word list recall, delayed recall, animal category fluency, and the symbol digit modalities test (all $p<0.01$ ) compared to controls. Conclusion: COPD is associated with a more rapid rate of cognitive decline in very elderly persons.

Copyright $\odot 2012$ S. Karger AG, Basel
\end{abstract}

\section{Introduction}

With an aging population on the rise worldwide and a shift from an acute to a chronic disease burden, the incidence and prevalence of cognitive impairment, such as Alzheimer's disease (AD) and vascular dementia (VD), are expected to increase in the coming decades 
$[1,2]$. Cognitive dysfunction is associated with increased mortality and disability, and maintenance of cognitive function is an important component for successful aging [3]. As a result, prevention of cognitive impairment through risk factor identification and modification has profound clinical significance [4]. Chronic obstructive pulmonary disease (COPD) is a multi-component disease that is common in the elderly $[5,6]$. In addition to these pulmonary abnormalities, COPD is also associated with significant effects in distant organs outside the lungs, the so-called extrapulmonary effects. These systemic effects add to the morbidity of affected patients and should be considered in the clinical assessment, as well as the treatment [7]. The majority of studies, but not all, have shown that patients with COPD have significant cognitive impairment, either globally or in domains, such as perception, memory, and psychomotor functions [8-16]. However, there have been few longitudinal studies examining the association of COPD with the course of cognitive decline. One recent population-based longitudinal study showed that severe COPD is associated with lower cognitive performance on standardized measurements over time [17]. Nonetheless, the clinical diagnosis of COPD and comorbidities based on self-report lack sensitivity and reliability, and applying cognitive screening instruments did not permit an in-depth examination of cognitive function. The participants of our study were retired male veterans with free medical care, similar social and educational backgrounds and lifestyle habits. These hospital-based participants provided an excellent opportunity to examine the change in cognitive function in the elderly. Our study evaluated cognitive performance in patients with COPD over a 3 -year period relative to control participants. We hypothesized that COPD is associated with accelerated cognitive decline.

\section{Methods}

\section{Study Settings}

All the participants were retired male military officers aged $\geq 75$ years and were registered at retirement. The cohort consisted of nearly 40 veteran convalescent camps in Nanjing, China, which serviced approximately 5,000 retired male military officers of high rank. Each camp had nurses and clinicians who cared for the elderly and interacted with physicians at the Jinling Hospital of the Nanjing University School of Medicine when necessary. All the elderly living in the long-term care system had received free medical examinations and treatment, as well as hospitalization in the Jinling Hospital. The retired male military officers underwent an overall physical examination once per year after which $>65 \%$ were hospitalized. The study was approved by the Institutional Review Board of the Jinling Hospital. All subjects signed an informed consent.

\section{Cases}

All the patients with COPD who were hospitalized in a 200-bed geriatric ward of the Jinling Hospital between January and December 2007 were systematically and prospectively screened for inclusion in our study irrespective of the indication for hospital admission. On admission, all patients underwent a routine medical examination, including standard blood tests, chest X-ray, electrocardiogram, brain MRI or CT scan, and ultrasound examination. The study physicians confirmed the diagnosis of COPD by applying standardized criteria to medical data, hospitalization records, and spirometry results (forced expiratory volume in $1 \mathrm{~s}$ [FEV1]/forced vital capacity [FVC] $<0.7$ and FEV1 $<80 \%$ of predicted). During hospitalization, the following clinical data were documented: demographic characteristics; medications used; history of heart disease (angina, myocardial infarction, atrial fibrillation, and chronic heart failure), hypertension, cancer, diabetes mellitus, and chronic 
renal disease. Depressive symptoms were assessed using the Hamilton Depression Scale. A global summary score on the Clinical Dementia Rating (CDR) was also assigned. Inclusion in the study required the following criteria: (1) patients had to be in a stable condition with no physical findings or symptoms suggestive of acute exacerbation before cognitive function testing, and (2) they had to be in a relatively healthy condition without oxygen dependence. The exclusion criteria included the following: (1) a diagnosis of major neurologic or psychotic disorders (e.g. Parkinson disease, dementia, clinical stroke, or depression); (2) taking medications with potentially potent central nervous system effects (e.g. sedatives or anti-psychotics); (3) considerable hearing or sight disabilities, and (4) major chronic medical illness that could affect cognitive function (cancer, diabetes mellitus, renal disease, atrial fibrillation, and chronic heart failure). Because hypertension and coronary heart disease (angina and myocardial infarction) are extremely common in elderly patients, they were not included in the exclusion criteria. The initial eligible cases included 113 elderly males.

Controls

Controls were selected from the elderly who were admitted for general health examinations in the same geriatric wards during the same study periods as the COPD patients. The clinical evaluation and detailed examination for the controls was the same as for the cases. The control subjects were matched for the following characteristics: (1) age within 1 year, (2) educational level, and (3) admission date within 30 days. Elderly subjects who were in a relatively good health status were included as controls. The exclusion criteria for the case group were also applied to the selection of the control group.

\section{Measurement of Cognitive Function}

Trained examiners carried out the following cognitive tests in a quiet room of a geriatric ward. We used the Chinese version of the Mini-Mental State Examination (MMSE) [18] to measure global cognitive functioning with summed scores ranging from 0 to 30 . For the word list recall (immediate recall) $[18,19]$, a 10-word list was presented and free recall assessed across three trials with the words being presented in a different order for each trial. The list was presented in random order on three successive occasions. After each occasion, the participant reported all the words he could recall. The scoring range on each trial was $0-10$; the total score was 30 for the three trials. The animal category fluency $[18,19]$ assesses language, verbal fluency, and executive functioning by asking the subject to name as many animals as possible within $1 \mathrm{~min}$. The test score was the total number of animals named. In the symbol digit modalities test $[18,19]$, the subject has to translate the numbers 1-9 into symbols within $90 \mathrm{~s}$. The test score is the total number of correct translations to symbols within the set time. Word delayed recall $[18,19]$ for the 10 words in the word list (immediate recall) test was assessed 5 min after the third trial on immediate memory, with a scoring range of $0-10$.

\section{Follow-Up}

Every participant was followed annually for 3 years after the initial cognitive performance. Because 2 patients with COPD and 1 control subject died before the first follow-up evaluation, 3 patients with COPD ( 2 COPD patient deaths and 1 patient matched for the control patient death) and 3 control participants ( 1 control patient death and 2 control patients matched for the 2 COPD patient deaths) were excluded from the study. Thus, 220 persons (110 patients and 110 control subjects) were eligible for follow-up and were used in the final analysis. During the 3 years of follow-up, the frequency and cause of hospitalizations for patients with COPD and control subjects were also recorded. 
Table 1. Baseline characteristics of the patients with COPD and the control group at baseline

\begin{tabular}{lccc}
\hline & $\begin{array}{l}\text { COPD } \\
(\mathrm{n}=110)\end{array}$ & $\begin{array}{l}\text { Control group } \\
(\mathrm{n}=110)\end{array}$ & p value \\
\hline $\begin{array}{l}\text { Age, years } \\
\text { Educational level, } \%\end{array} \quad 80.9 \pm 1.78$ & $80.8 \pm 1.59$ & 0.720 \\
$\quad$ 5 years & $17(15.5)$ & $17(15.5)$ & matched \\
$\quad 72$ years & $72(65.5)$ & $72(65.5)$ & matched \\
$\quad 210$ years & $21(19.1)$ & $21(19.1)$ & 0.225 \\
BMI & $81(73.6)$ & $22.8 \pm 2.7$ & 0.023 \\
Hypertension & $54(49.1)$ & $64(58.2)$ & 0.040 \\
Coronary heart disease & $27.1 \pm 1.67$ & $27.3 \pm 1.59$ & 0.458 \\
MMSE & $16.1 \pm 2.89$ & $17.0 \pm 2.61$ & 0.018 \\
Word list recall & $5.2 \pm 1.18$ & $5.5 \pm 1.29$ & 0.116 \\
Delayed recall & $14.2 \pm 2.09$ & $14.8 \pm 2.25$ & 0.022 \\
Animal fluency & $23.1 \pm 5.26$ & $24.6 \pm 3.76$ & 0.014 \\
Symbol digit & & & \\
\hline
\end{tabular}

Data are presented as the mean \pm SD or $\mathrm{n}(\%)$.

$p$ values were determined using a paired $t$ test and the $\chi^{2}$ analysis.

\section{Statistical Analysis}

We used a series of linear mixed-effects models to examine the effects of COPD at baseline and the annual rate of change (fixed effects) in cognition during the study period, while adjusting for person-specific paths of change with random effects. In this growth curve approach, the path of each individual is assumed to follow the mean path of the group, except for random effects that cause the initial level of function (i.e. intercept) to be higher or lower and the rate of change (i.e. slope) to be faster or slower. We constructed separate mixed-effects models for each of the cognitive test scores. Each model included terms for time (years since baseline), the presence of COPD, and the interaction. The term for time indicates the average annual change in the score for control subjects. The term for COPD denotes the average difference in the baseline score between patients with COPD and the control group. The interaction term $(\mathrm{COPD} \times$ time $)$ indicates the average additional annual change in the score associated with having COPD. All analyses were adjusted for hypertension and coronary heart disease. Statistical analyses were performed using Stata 10 (StataCorp., College Station, Tex., USA). All reported p values were two-sided, and the results were considered statistically significant at $\mathrm{p}<0.05$.

\section{Results}

\section{Baseline Characteristics of the Two Groups}

The baseline characteristics of the elderly with COPD and the controls were well-matched for age, education and body mass index (BMI) at baseline, but there were more patients with hypertension $(\mathrm{p}=0.023)$ and coronary heart disease $(\mathrm{p}=0.04)$ among those with COPD compared to controls. The COPD patients had poorer baseline cognitive scores on the word list recall $(\mathrm{p}=0.018)$, animal fluency $(\mathrm{p}=0.022)$, and symbol digit tests $(\mathrm{p}=0.014)$, but the scores of MMSE and delayed recall were not different between the two groups ( $\mathrm{p}>0.05$; table 1). 
Table 2. Summary of mixed-effect models examining the relationship of COPD to the baseline level and annual rate of change in different cognitive domains

\begin{tabular}{|c|c|c|c|c|c|c|c|c|c|}
\hline & \multicolumn{3}{|l|}{ COPD } & \multicolumn{3}{|l|}{ Time } & \multicolumn{3}{|c|}{$\mathrm{COPD} \times$ time } \\
\hline & $\mathrm{B}$ & SE & $\mathrm{p}$ value & B & SE & $\mathrm{p}$ value & B & SE & $\mathrm{p}$ value \\
\hline MMSE & -0.221 & 0.218 & 0.313 & -0.238 & 0.0304 & $<0.001$ & -0.219 & 0.0413 & $<0.001$ \\
\hline Word list recall & -0.874 & 0.364 & 0.016 & -0.265 & 0.0277 & $<0.001$ & -0.224 & 0.0391 & $<0.001$ \\
\hline Delayed recall & -0.259 & 0.161 & 0.107 & -0.159 & 0.0193 & $<0.001$ & -0.077 & 0.0274 & $<0.005$ \\
\hline Animal fluency & -0.659 & 0.294 & 0.024 & -0.238 & 0.0295 & $<0.001$ & -0.231 & 0.0417 & $<0.001$ \\
\hline Symbol digit & -1.484 & 0.609 & 0.015 & -0.354 & 0.0327 & $<0.001$ & -0.267 & 0.0467 & $<0.001$ \\
\hline
\end{tabular}

Follow-Up

During the follow-up, 209 subjects (95\%) completed two reassessments of cognitive function, and 205 subjects (93\%) completed three reassessments of cognitive function. During the 3 years of follow-up, the mean frequency of hospitalization per subject did not differ significantly between the case and control groups $(2.9 \pm 1.4$ vs. $2.7 \pm 1.2, \mathrm{p}=0.152)$. However, the principal indication for hospital admission (85\% of the total number of hospitalizations) for the COPD group was exacerbation of COPD as a primary diagnosis, while for control subjects, completion of a routine physical examination and common chronic complaints (dizziness or fatigue) were the most common indications for hospital admissions ( $83 \%$ of the total number of hospitalizations).

\section{Relationship between COPD and Level of and Change in Cognitive Tests}

We examined the associations between cognition in the COPD patients at baseline and the annual decline in cognitive scores using a mixed-effects model that controls for hypertension and coronary heart disease (table 2). At baseline, subjects with COPD had lower scores than controls on the word list recall $(\mathrm{p}=0.016)$, animal fluency $(\mathrm{p}=0.024)$, and symbol digit tests $(\mathrm{p}=0.015)$, as shown by the term for COPD, although the COPD patients had similar baseline scores on MMSE and delayed recall compared with the control group ( $\mathrm{p}>$ $0.05)$. Control subjects had a mean decline in the MMSE score of 0.238 points/year ( $\mathrm{p}<$ $0.001), 0.265$ points/year on the word list recall $(\mathrm{p}<0.001), 0.159$ points/year on the delay recall ( $\mathrm{p}<0.001), 0.238$ points/year on animal fluency $(\mathrm{p}<0.001)$, and 0.354 points/year on the symbol digit tests $(\mathrm{p}<0.001)$, as shown by the term for time. Compared with the control subjects, the cognitive scores in patients with COPD declined by an additional 0.219 points/ year for the MMSE $(\mathrm{p}<0.001)$, an additional 0.224 points/year for the word list recall $(\mathrm{p}<$ $0.001)$, an additional 0.077 points/year for delayed recall $(\mathrm{p}=0.005)$, an additional 0.231 points/year for animal fluency $(\mathrm{p}<0.001)$, and an additional 0.267 points/year for the symbol digit tests $(\mathrm{p}<0.001)$, as shown by the interaction term $(\mathrm{COPD} \times$ time $)$. Cognitive functions of the patients with COPD and the control group at baseline and at the 3-year follow-up in different domains are shown in figure 1.

\section{Discussion}

In this hospital-based, prospective case-control study, we found that COPD was related to worse cognitive function at baseline and cognitive decline on the word list recall, animal category fluency, and symbol digit modalities over a 3-years observation period. Despite a 

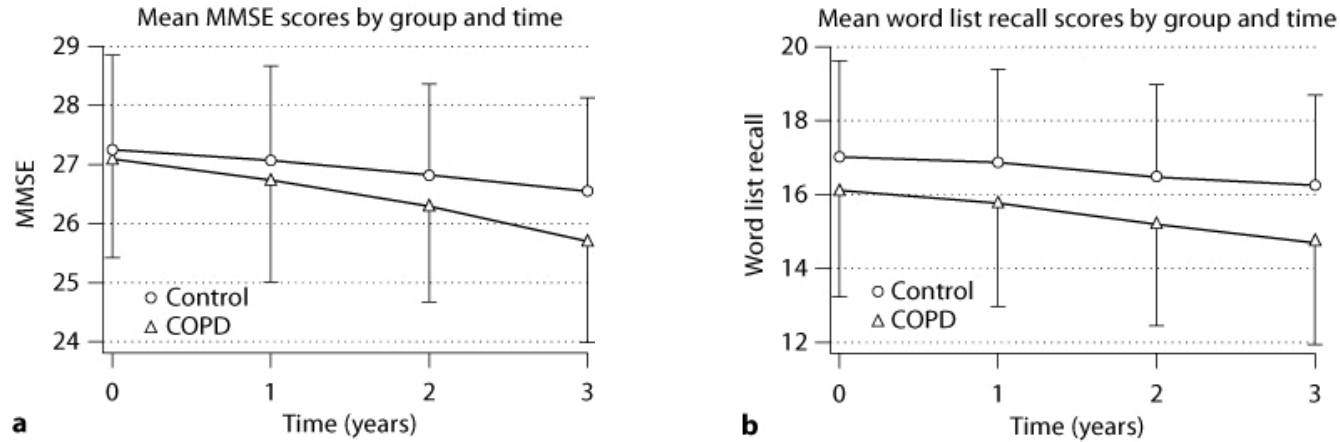

Mean delayed recall scores by group and time
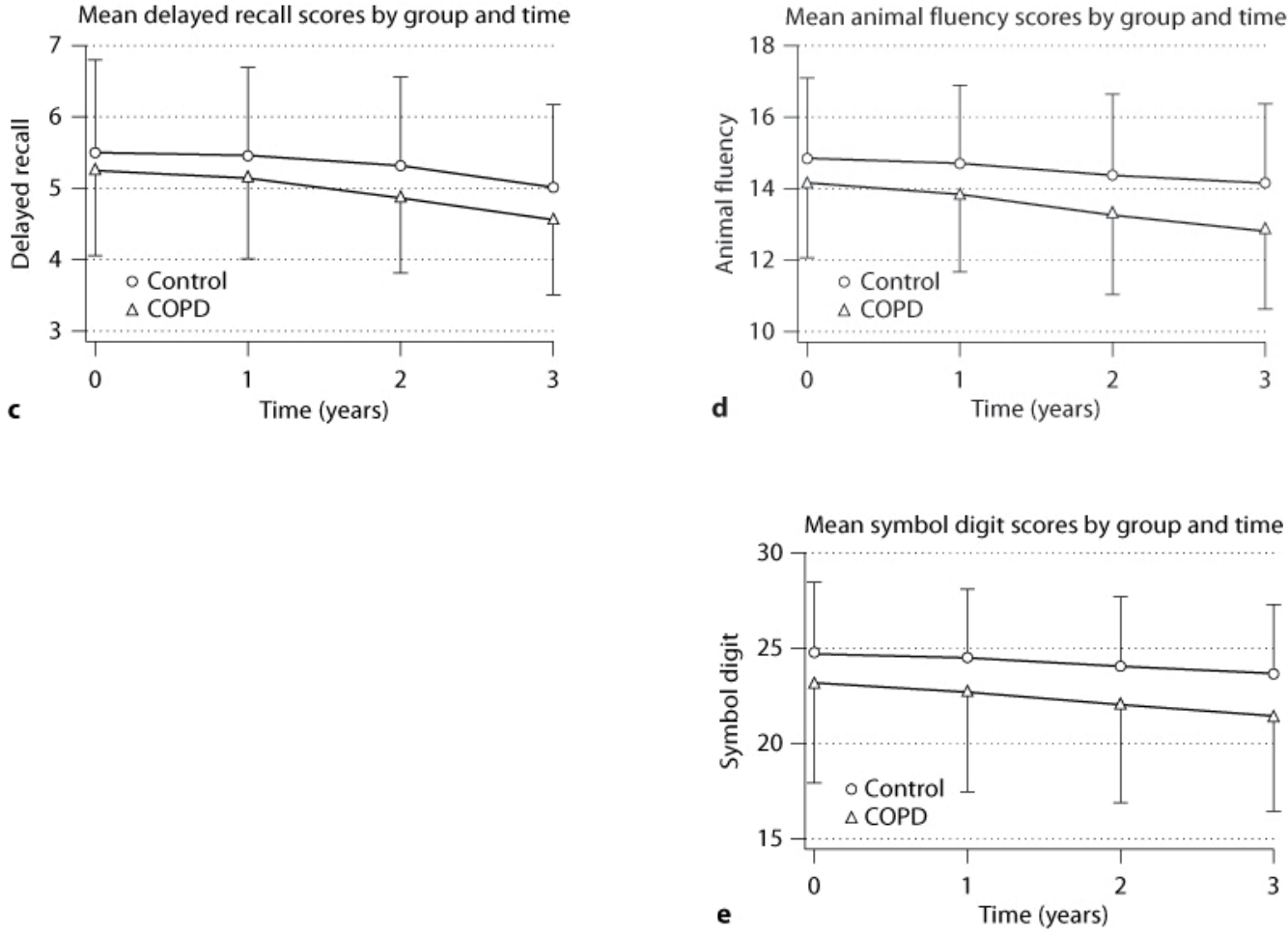

Fig. 1. Cognitive functions of the patients with COPD and the control group at baseline and at the 3-year follow-up in different domains.

lack of association between COPD with the scores of MMSE and delayed recall at baseline, persons with COPD still had significant declines in mean scores of MMSE and delayed recall compared with control participants. Our findings extend the findings of a previous crosssectional study, and suggest that COPD is a significant risk factor for cognitive decline in older persons.

Since the 1970s, cognitive performance has been studied in patients with COPD. The majority of studies have shown that patients with COPD have a higher rate of cognitive impairment, either globally or in perception, memory, and psychomotor function domains 
[8-16]. However, longitudinal studies that have examined the decline in the cognitive function profile of patients with COPD and control participants by means of detailed cognitive tests are limited. One recent study which included 4,150 adults with a mean age of 62 years showed that severe COPD is associated with poorer cognitive performance on standardized measurements over time [17]. However, it was noted that the diagnosis of COPD and other comorbidities was based on self-report. Moreover, applying cognitive screening instruments does not allow for an analysis of the relationship between COPD and specific cognitive domains. It is increasingly recognized that dementia may better be interpreted as a continuum of symptoms, rather than the mere presence or absence of a disease. Because cognitive decline or dementia develops over a long preclinical period, it is difficult to reliably identify the factors which influence cognitive decline. A previous study suggested that timing is important in the association between the factors which influence cognitive decline or dementia [20]. For example, a higher BMI at baseline was shown to be associated with a higher cognitive decline in middle-aged men and women during follow-up [21], while a low BMI in late life may be a preclinical indicator of underlying dementia [22]. COPD has a deleterious effect on cognitive function, but the degree and range of the effect may relate to the age of patients with COPD. Because very elderly persons may be particularly vulnerable to pathologic processes or diseases that cause cognitive decline, our participants with a mean age of $>80$ years were likely to have a rapid and more diffuse pattern of cognitive decline over a 3-year period. To limit the possibility that individuals with cognitive impairment would affect the association between COPD and cognitive decline, our sample only included individuals in relatively good health at baseline. On the basis of a series of medical and laboratory examinations, as well as a summary score on the Clinical Dementia Rating, all the participants were considered to be cognitively normal elderly. Furthermore, as very elderly persons inevitably have a higher prevalence of chronic disease and disability, which may relate to cognitive decline, participants with severe chronic disease were also excluded from the current study. Although there were still more COPD patients with hypertension and coronary heart disease than controls, in this age- and education-matched case-control study, the baseline characteristics were well-balanced between the two groups. The more stringent inclusion criteria in our study may avoid the influence of possible confounding variables, therefore our results more likely reflect the effect of COPD on cognitive decline in very elderly persons using a mixed model adjusted for hypertension and coronary heart disease.

A key mechanism proposed for cognitive dysfunction in COPD is neuronal damage mediated by hypoxia [23]. For example, hypoxia might cause changes in ionic channels, signaling pathways, neuromodulators, and genomic processes; low acetylcholine concentrations have been found in the neocortex and hippocampus [24, 25]. However, the correlation between cognitive function and arterial oxygen tension is weak, and oxygen therapy of longer duration has shown no benefit of cognitive improvement in hypoxemic individuals $[23,26]$. Current evidence suggests that hypoxemia alone is not enough to entirely account for the cognitive deficits which occur in COPD. The mechanisms of cognitive decline underlying COPD are probably interrelated and multifactorial, including tissue hypoxia, systemic inflammation, and oxidative stress $[23,25]$. Because the elderly who have undergone annual routine health examinations usually prefer to be admitted to the hospital, the frequency of hospitalization between the case and control groups did not differ significantly. However, acute exacerbation is the major cause of hospitalization in patients with COPD. Some studies have directly addressed the deleterious impact of COPD exacerbations on cognitive function $[23,27]$. Recently, a study showed that older adults who had acute care and critical illness hospitalizations had a greater likelihood of cognitive decline compared with older adults who were not hospitalized, suggesting that factors associated with acute illness may be causally related to cognitive decline [28]. COPD exacerbations are a common cause of acute hos- 
pitalization. The mechanisms through which COPD exacerbation contributes to cognitive decline may be multiple, including hypoxemia, hypotension, systemic inflammation, and medications. Given that the elderly with COPD were in relatively good health and without oxygen dependence, we emphasize that the high frequency of COPD exacerbations, which trigger a complicated pathologic process, rather than chronic hypoxia only, may have contributed to cognitive decline in this study.

Our study employed a brief cognitive screening test and three domain-specific cognitive tests. The animal fluency is a sensitive test for detecting frontal lobe damage with deficits in executive functioning. The symbol digit modalities test is a measure of psychomotor speed and executive function and has been demonstrated to show impairment in VD. Word lists with immediate and delayed recall are sensitive to memory loss associated with an early stage of $\mathrm{AD}$. In order of prevalence, $\mathrm{AD}$ and VD are the most common causes of dementia in elderly people, and subcortical ischemic vascular dementia (SIVD), due to small vessel disease, is clinically homogeneous and a major cause of vascular cognitive impairment and VD [29, 30]. Typically, memory impairment resulting from atrophy in the medial temporal lobe is the first sign of $A D$, while SIVD is characterized by deficits in attention, executive function, psychomotor speed, and information processing with milder memory loss as compared with $\mathrm{AD}$. The majority of the literature concerning cognitive impairment in patients with COPD points to the subcortical type with a predominant decline in attention, slower mental speed, and executive function [25]. These findings are consistent with low oxygen saturation and COPD being associated with more severe white matter periventricular lesions [31]. Our study revealed that COPD was related to cognitive decline in multiple cognitive domains, either globally or in domains, such as memory, psychomotor speed, and executive function. Given the fact that participants with large cerebrovascular lesions/clinical stroke were excluded after detailed clinical examinations and brain CT scans, this non-specific pattern of cognitive decline resembles mixed SIVD/AD. The result reinforces the recent idea that mixed pathologies of vascular and neurodegenerative brain injuries most likely represent the majority of elderly with cognitive decline $[32,33]$. Because dementia was not formally diagnosed at the end of follow-up, there may be the possibility that a few of the participants in the current study were in an early stage of dementia. Furthermore, continued follow-up may help to understand the relationship between COPD and frank dementia.

Our study had some limitations. First, our sample size was small, and the probability estimates were derived from a case-control study with the inherent weaknesses of selection bias. Although participants were stringently selected to avoid the influence of possible confounding variables, such as diabetes, cerebrovascular disease, and major chronic diseases, there is a possibility that other chronic or subclinical diseases which were not included in the analysis may also have contributed to cognitive decline. Second, we do not have measures of inflammatory markers related to COPD exacerbations and cognitive function, and we did not examine the effect of COPD severity and exacerbations on the relationship between COPD and cognitive decline. Finally, our sample consists of elderly men only, so the data may not be applicable to other data or populations with a different socioeconomic background. However, confidence in the findings of this study was strengthened by the following factors: cognitive performance in the hospital ward administered by specially trained examiners; availability of detailed medical records and annual hospital observations with a high rate of follow-up, and the homogeneity of our sample which may control the effects of potentially confounding variables, such as lifestyle and occupation.

In summary, these results indicate that very elderly men with COPD have a more rapid and diffuse pattern of cognitive decline over a 3-year period compared with age- and education-matched control participants. 


\section{References}

-1 Kalaria RN, Maestre GE, Arizaga R, Friedland RP, Galasko D, Hall K, et al: Alzheimer's disease and vascular dementia in developing countries: prevalence, management, and risk factors. Lancet Neurol 2008;7:812-826.

-2 Pendlebury ST, Rothwell PM: Prevalence, incidence, and factors associated with pre-stroke and poststroke dementia: a systematic review and meta-analysis. Lancet Neurol 2009;8:1006-1018.

-3 Fiocco AJ, Yaffe K: Defining successful aging. Arch Neurol 2010;67:876-880.

4 Middleton LE, Yaffe K: Promising strategies for the prevention of dementia. Arch Neurol 2009;66: $1210-1215$.

-5 Durme YM, Verhamme KM, Stijnen T, Rooij FJ, Pottelberge GR, Hofman A, et al: Prevalence, incidence, and lifetime risk for the development of COPD in the elderly: the Rotterdam study. Chest 2009; 135:368-377.

-6 Viegi G, Pistelli F, Sherrill DL, Maio S, Baldacci S, Carrozzi L: Definition, epidemiology and natural history of COPD. Eur Respir J 2007;30:993-1013.

7 Agustif AG: Systemic effects of chronic obstructive pulmonary disease. Proc Am Thorac Soc 2005; 2:367-370.

8 Grant I, Heaton RK, McSweeny AJ: Neuropsychologic findings in hypoxemic chronic obstructive pulmonary disease. Arch Intern Med 1982;142:1470-1476.

-9 Antonelli-Incalzi R, Corsonello A, Trojano L: Screening of cognitive impairment in chronic obstructive pulmonary disease. Dement Geriatr Cogn Disord 2007;23:264-270.

-10 Incalzi RA, Gemma A, Marra C: Chronic obstructive pulmonary disease. An original model of cognitive decline. Am Rev Respir Dis 1993;148:418-424.

-11 Fix AJ, Golden CJ, Daughton D: Neuropsychological deficits among patients with chronic obstructive pulmonary disease. Int J Neurosci 1982;16:99-105.

-12 Incalzi RA, Gemma A, Marra C: Verbal memory impairment in COPD: its mechanisms and clinical relevance. Chest 1997;112:1506-1513.

-13 Favalli A, Miozzo A, Cossi S: Differences in neuropsychological profile between healthy and COPD older persons. Int J Geriatr Psychiatry 2008;23:220-221.

-14 Liesker JW, Postma DS, Beukema RJ, ten Hacken NH, van der Molen T, Riemersma RA, et al: Cognitive performance in patients with COPD. Respir Med 2004;98:351-356.

-15 Incalzi RA, Corsonello A, Pedone C, Trojano L, Acanfora D, Spada A, et al: Drawing impairment predicts mortality in severe COPD. Chest 2006;130:1687-1694.

-16 Salik Y, Ozalevli S, Cimrim AH: Cognitive function and its effects on the quality of life status in the patients with chronic obstructive pulmonary disease. Arch Gerontol Geriatr 2007;45:273-280.

-17 Hung WW, Wisnivesky JP, Siu AL, Ross JS: Cognitive decline among patients with chronic obstructive pulmonary disease. Am J Respir Crit Care Med 2009;180:134-137.

18 Tang CM: Neuropsychology, Beijing, People's Military Press (in Chinese), 2002.

19 Spreen O, Strauss E: A compendium of neuropsychological test, ed 2, New York, Oxford University Press, 1998.

20 Rocca WA, Grossardt BR, Shusterd LT: Oophorectomy, menopause, estrogen, and cognitive aging: the timing hypothesis. Neurodegener Dis 2010;7:163-166.

-21 Cournot M, Marquie JC, Ansiau D, Martinaud C, Fonds H, Ferrieres J, et al: Relation between body mass index and cognitive function in healthy middle-aged men and women. Neurology 2006;67: $1208-1214$.

-22 Hughes TF, Borenstein AR, Schofield E, Wu Y, Larson EB: Association between late-life body mass index and dementia. Neurology 2009;72:1741-1746.

-23 Dodd JW, Getov SV, Jones PW: Cognitive function in COPD. Eur Respir J 2010;35:913-922.

-24 Patel AJ, Honore E: Molecular physiology of oxygen-sensitive potassium channels. Eur Respir J 2001; $18: 227-233$

25 Areza-Fegyveres R, Kairalla RA, Carvalho CR, Nitrini R: Cognition and chronic hypoxia in pulmonary diseases. Dement Neuropsychol 2010;4:14-22.

-26 Pretto JJ, Mcdonald CF: Acute oxygen therapy does not improve cognitive and driving performance in hypoxaemic COPD. Respirology 2008;13:1039-1044. 
-27 Kirkil G, Tug T, Ozel E: The evaluation of cognitive functions with P300 test for chronic obstructive pulmonary disease in attack and stable period. Clin Neurol Neurosurg 2007;109:553-560.

- 28 Ehlenbach WJ, Hough CL, Crane PK, Haneuse SA, Carson SS, Curtis JR, et al: Association between acute care and critical illness hospitalization and cognitive function in older adults. JAMA 2010;303: 763-770.

-29 Viswanathan A, Rocca WA, Tzourio C: Vascular risk factors and dementia: how to move forward. Neurology 2009;72:368-374.

-30 Jokinen H, Kalska H, Mantyla R, Pohjasvaara T, Ylikoski R, Hietanen M, et al: Cognitive profile of subcortical ischaemic vascular disease. J Neurol Neurosurg Psychiatry 2006;77:28-33.

-31 van Dijk EJ, Vermeer SE, de Groot JC: Arterial oxygen saturation, COPD, and cerebral small vessel disease. J Neurol Neurosurg Psychiatry 2004;75:733-736.

-32 Roman GC, Erkinjuntti T, Wallin A, Pantoni L, Chui HC: Subcortical ischaemic vascular dementia. Lancet Neurol 2002;1:426-436.

-33 Strozyk D, Dickson DW, Lipton RB, Katz M, Derby CA, Lee S, et al: Contribution of vascular pathology to the clinical expression of dementia. Neurobiol Aging 2010;31:1710-1720. 